\title{
Dispersão de partículas em cabine de aeronave com insuflamento de ar pelo teto e pelo piso
}

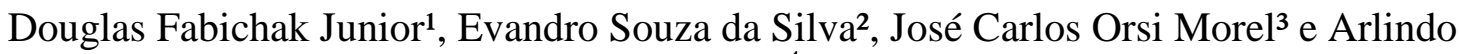 \\ Tribess $^{4}$ \\ Escola Politécnica da Universidade de São Paulo \\ E-mails: fabichak@ usp.br, evandrosouzadasilva@yahoo.com.br, metrofis@ gmail.com, \\ atribess@usp.br
}

\section{RESUMO}

Sistemas de ventilação comumente utilizados em cabines de aeronaves consistem no insuflamento de ar na parte superior, pelo teto, e retorno na parte inferior, com mistura do ar na cabine (sistema MV). Devido à sua característica de mistura, este sistema pode espalhar doenças infecciosas pelo ar na cabine. A eclosão mundial do vírus SARS (Severe Acute Respiratory Syndrome) em 2003 demonstrou que a disseminação de contaminantes aéreos ainda é um evento não controlável, uma vez que foi rapidamente difundido mundo afora, principalmente porque pessoas infectadas viajaram de avião para cidades distantes. Fatos como esses têm motivado governos, empresas e instituições de pesquisa a investirem fortemente em pesquisa e desenvolvimento. Novos sistemas de ventilação e de distribuição de ar em aeronaves, baseados em sistemas de ventilação por deslocamento (DV) e sistemas de ventilação pelo piso (UFAD), estão começando a ser estudados. O objetivo deste trabalho é realizar análise experimental de dispersão de partículas, considerando sistema de ventilação pelo teto (MV) e pelo piso (UFAD), em mock-up de cabine de aeronave de 12 lugares, utilizando gerador e contadores de partículas. O estudo foi focado na dispersão de partículas expiratórias geradas por pessoas. Os resultados de dispersão de partículas na cabine mostraram grande influência do sistema de ventilação e do local na aeronave onde as partículas são geradas pelas pessoas, como em um espirro, por exemplo. Verificou-se que o sistema pelo piso, UFAD, permite um maior controle na remoção e dispersão de partículas e, com isto, um maior controle na dispersão de contaminantes no ar da cabine.

\section{INTRODUÇÃO}

O ambiente de cabine de aeronaves é único quando comparado com outros tipos de veículos automotivos e de edificações, com exposição dos passageiros a um ambiente com umidade relativa muito baixa, por longo tempo, e sem possibilidade de sair da cabine.

O espaço interno é limitado e possui um número elevado de ocupantes, fazendo com que os passageiros fiquem muito próximos uns aos outros. Este fato, aliado a baixas taxas de ventilação por pessoa e o longo tempo de exposição, torna o ambiente favorável à transmissão de doenças infecciosas por contato e por partículas aéreas [1].

A eclosão mundial do vírus SARS (Severe Acute Respiratory Syndrome) em 2003 demonstrou que a disseminação de contaminantes aéreos ainda é um evento não controlável, uma vez que 
foi rapidamente difundido mundo afora, principalmente porque pessoas infectadas viajaram de avião para cidades distantes [2].

Mangili e Geandreau [3] citam vários casos de disseminação de enfermidades em cabines de avião, dentre elas as do vírus influenza, malária e dengue, mostrando que as cabines de aeronaves são ambientes propícios para a infecção cruzada de doenças.

Função disso, estudos de contaminação em cabines de aeronaves tem sido focados na contaminação aérea resultante da dispersão de poluentes expiratórios gerados por pessoas infectadas contaminando outras pessoas - a contaminação cruzada [1,4],[5-9].

\subsection{Sistemas de ventilação em cabines de aeronaves}

O ambiente de cabine de aeronave é regulado por um sistema de controle ambiental que controla a pressurização, a temperatura, a ventilação e a filtração do ar da aeronave.

O sistema de ventilação mais utilizado em cabines de aeronaves consiste no insuflamento de ar na parte superior, pelo teto, e retorno na parte inferior com mistura do ar na cabine, Mixing Ventilation - MV, Figura 1. O resultado é uma temperatura quase uniforme na cabine e grande dispersão de contaminantes.

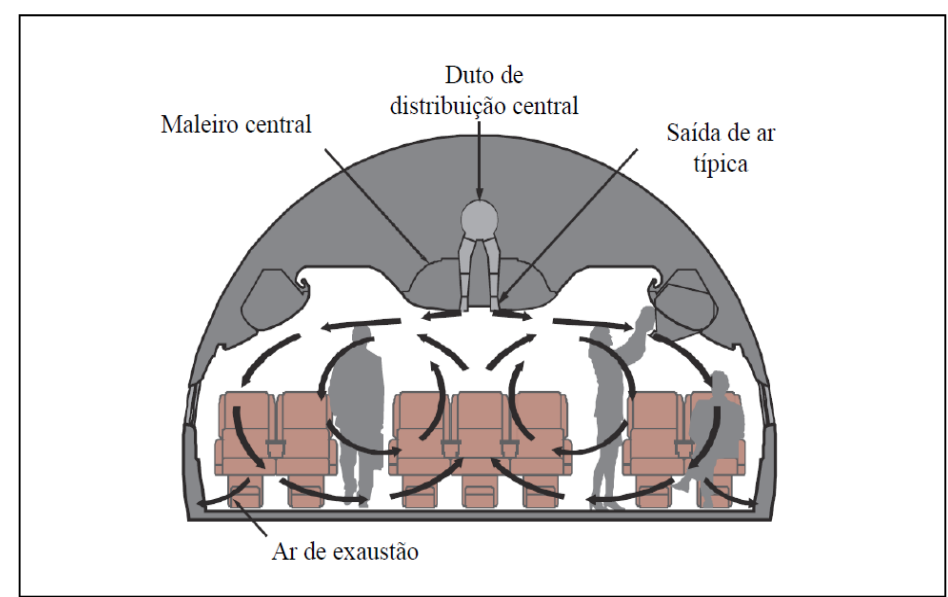

Figura 1. Sistema de ventilação com mistura de ar na cabine (adaptado [10]).

Embora o sistema MV, normalmente, proporcione um ambiente com baixa estratificação de temperatura, problemas de conforto térmico têm sido verificados. Além disso, devido à sua característica de mistura, pode espalhar mais facilmente doenças infecciosas pelo ar na cabine ([11] e [12]).

Para tentar resolver os problemas de conforto térmico e de qualidade do ar, novos sistemas de ventilação e de distribuição de ar em aeronaves estão começando a ser estudados [13] e testados [14], baseados em sistemas de ventilação por deslocamento, Displacement Ventilation - DV [15] e sistemas de ventilação pelo piso, UnderFloor Air Distribution UFAD [16], já aplicados em edificações. 
No sistema UFAD, ar fresco é insuflado no ambiente a partir do piso (Fig. 2), e de maneira semelhante ao sistema DV, o escoamento se soma à pluma térmica dos ocupantes levando os contaminantes até a exaustão pelo teto [17] e [18].

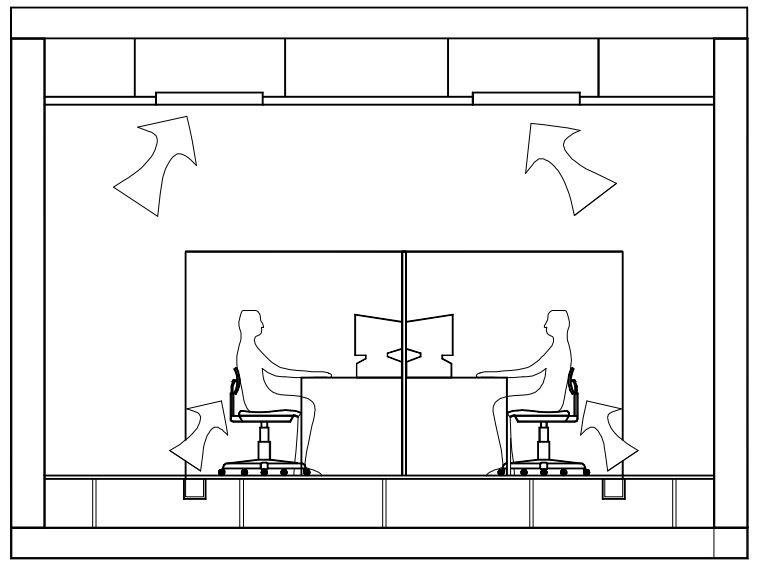

Figura 2. Sistema de ventilação pelo piso - UFAD.

1.2 Estudos de ventilação e de dispersão de partículas em cabines de aeronaves

Estudos experimentais têm sido realizados em mock-ups de aeronaves de uma seção da cabine ou em aeronaves paradas simulando condições de escoamento em voo. Isto ocorre porque estudos experimentais em condições reais de voo, quando conduzidos com razoável resolução espacial para obtenção de resultados confiáveis, são extremamente caros [13].

Wan et al., [4], realizaram estudo experimental de dispersão de gotículas expiratórias em mock-up com 21 assentos (três fileiras de sete assentos). O mock-up, instalado no International Center for Indoor Environment and Energy, da Dinamarca, é dotado de sistema de ventilação convencional, sistema MV, com pessoas sendo simuladas com a utilização de manequins aquecidos. No trabalho de Wan et al.[4], foi utilizado gerador de partículas simulando pessoa tossindo, desenvolvido pelos próprios pesquisadores. A Figura 3 mostra os pontos de injeção e de medição para duas vazões de ar de cabine de $100 \mathrm{~L} / \mathrm{s}$ e de $200 \mathrm{~L} / \mathrm{s}$, com medições realizadas a 1,10 m do piso (zona de respiração).
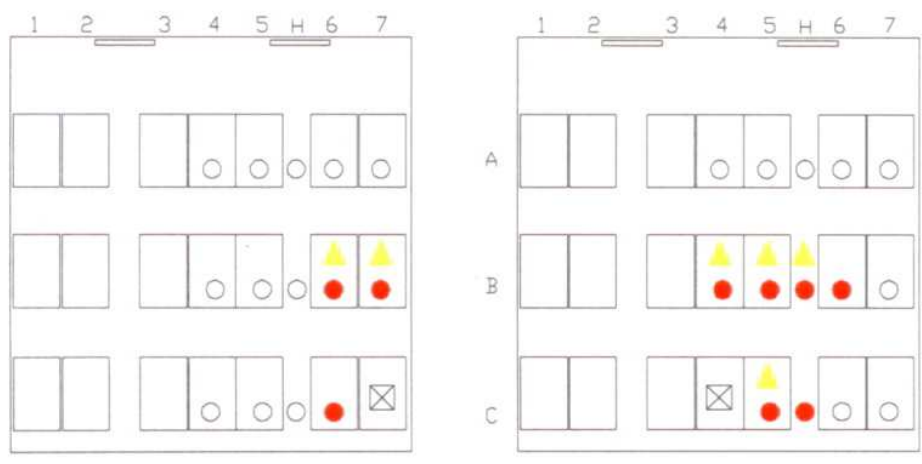

$\triangle$ Detecção a $200 \mathrm{~L} / \mathrm{s}$

Detecção a 100 L/s

Pontos de medição

Pontos de injeção

Figura 3. Pontos de injeção e de detecção de gotículas para diferentes vazões de ar de cabine [4]. 
Os resultados de Wan et al., [4], mostraram que um menor número de partículas foi disperso para outros assentos quando a injeção das partículas foi feita simulando passageiro junto à fuselagem em comparação com passageiro sentado em poltrona no centro do mock-up. Segundo os autores, isso sugere que o fluxo de ar descendente junto à fuselagem tenha suprimido a dispersão de partículas, enquanto o fluxo ascendente no centro do mock-up tenha aumentado à dispersão.

Zhang et al., [5], também estudaram a dispersão de partículas em mock-up de avião, patrocinado pelo FAA - Federal Aviation Authority - com manequins aquecidos do tipo caixa (Fig. 4) e sistema de ventilação convencional - sistema MV, focando nos campos de velocidade, temperatura e concentração de contaminantes. O objetivo do trabalho foi à validação e avaliação de modelos numéricos.

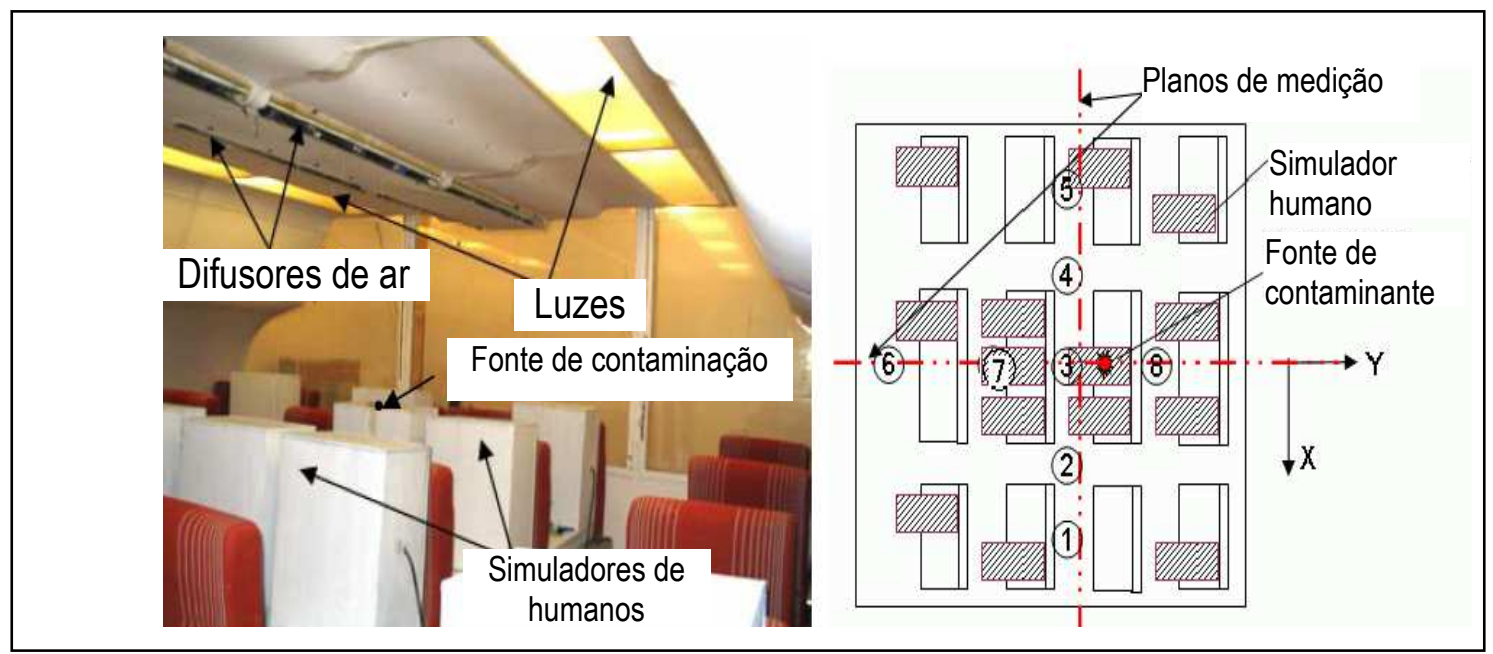

Figura 4. Mock-up de cabine de aeronave e planos de medição [5].

Yan et al., [7], estudaram a transmissão de contaminantes gerados por atividades expiratórias em mock-up de Boeing 737-300 (Fig. 5), também dotado de sistema convencional de ventilação, sistema MV. Os pesquisadores injetaram 4,5L/min de gás traçador $\left(\mathrm{CO}_{2}\right)$ nas proximidades da zona de respiração de um passageiro infectado durante 5 minutos, e a partir deste momento, monitoraram a concentração de $\mathrm{CO}_{2}$ na zona de respiração dos outros passageiros mediante a utilização de 14 sensores de $\mathrm{CO}_{2}$ por infravermelho não dispersivo. Os autores também realizaram simulação numérica utilizando dinâmica dos fluidos computacional - CFD (Computational Fluid Dynamics). De maneira geral, os autores concluíram que o sistema de ventilação influencia significativamente no mapa de concentração de partículas na cabine.

$\mathrm{Na}$ mesma linha de trabalho de Wan et al., [4], e utilizando as mesmas instalações e equipamentos do International Center for Indoor Environment and Energy, da Dinamarca, Sze To et al., [6], tiveram por objetivo caracterizar a dispersão e deposição de gotículas provenientes de atividades expiratórias em cabine de aeronaves, bem como identificar os efeitos do sistema global de ventilação, sistema MV, na diluição e remoção dessas partículas.

Os resultados obtidos por Sze To et al., [6], mostraram que o aumento da vazão de ar de renovação (maior vazão) provoca redução na concentração de partículas nas proximidades do ponto de injeção, devido ao efeito de diluição. Porém, a dispersão de partículas aumentou, o 
que provocou uma elevação da concentração de contaminantes nos assentos mais distantes do ponto de injeção. Foi observado também que a dispersão das partículas depende do seu tamanho médio, tendo sido obtidos índices de deposição de partículas da ordem de 60 a $70 \%$. Neste estudo também não foi investigada a influência de diferentes formas de ventilação na dispersão dos contaminantes na cabine.

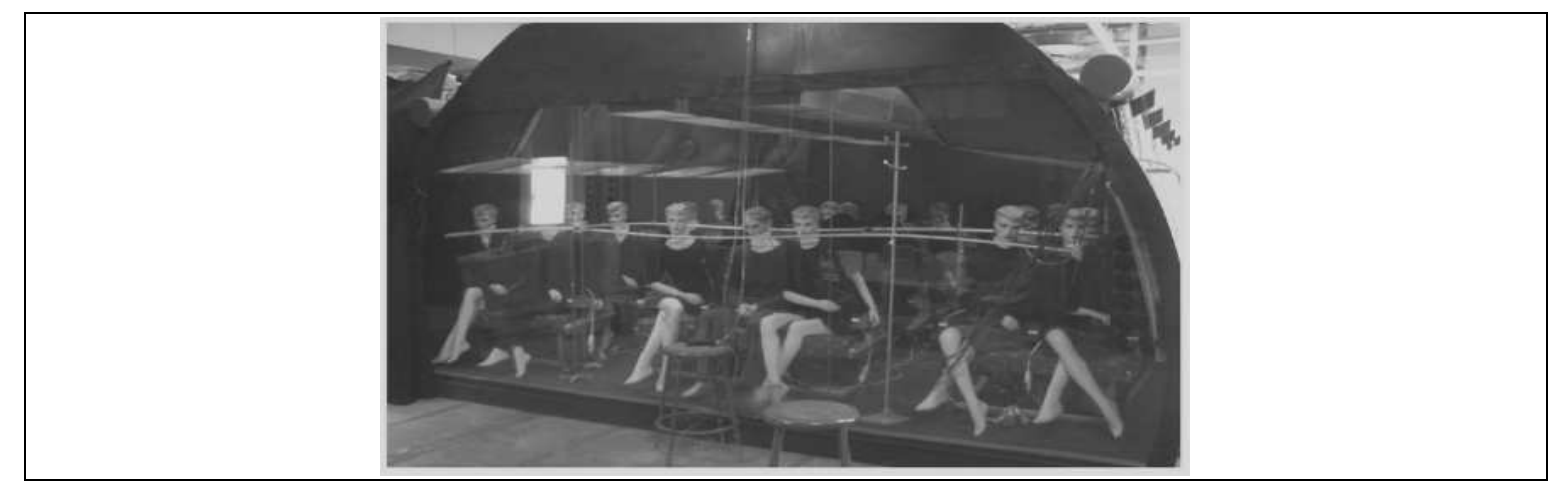

Figura 5. Estudo da dispersão de contaminantes em mock-up do Boeing 767-300

[7].

Observa-se nos estudos até aqui apresentados que todos tratam da análise experimental da contaminação aérea em sistema de ventilação por mistura, sistema $\mathrm{MV}$, pelo teto, que é o sistema utilizado nas aeronaves comerciais atualmente. Não foram encontrados na literatura aberta estudos experimentais com outros sistemas de ventilação e distribuição de ar. Foi encontrado somente um trabalho de simulação CFD, o trabalho de Zhang e Chen, [13], que realizaram estudos da dispersão de $\mathrm{CO}_{2}$ gerado pela respiração de passageiros em cabine de B767-300, com três formas de insuflamento de ar: ventilação por mistura - MV, ventilação pelo piso - UFAD, e ventilação pelo piso juntamente com ventilação personalizada.

Recentemente, Conceição, [9], desenvolveu trabalho de doutorado na análise experimental e numérica (CFD) de contaminação cruzada em mock-up de aeronave de 20 lugares (Fig. 6), sistema convencional MV, instalado no Laboratório de Conforto Térmico e Qualidade do Ar do Departamento de Engenharia Mecânica da Escola Politécnica da Universidade de São Paulo.

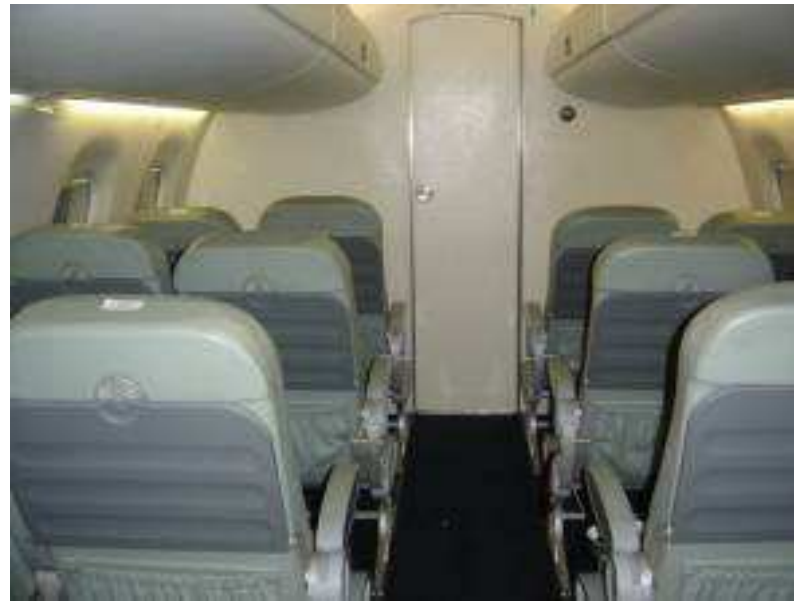

Figura 6. Mock-up de 20 lugares utilizado no desenvolvimento do trabalho [9]. 
No seu trabalho, Conceição, [9], desenvolveu processo de avaliação de dispersão de partículas em cabines climatizadas, que foi testado na análise da influência de sistema de ventilação personalizado (válvula gasper) no mock-up. O processo de avaliação desenvolvido no trabalho de Conceição, [9], foi utilizado no presente trabalho.

\section{OBJETIVO}

Este artigo tem como objetivo realizar análise experimental de dispersão de partículas em cabine de aeronave considerando dois sistemas distintos de ventilação: pelo teto (MV) e pelo piso (UFAD), em mock-up de 12 lugares, utilizando gerador e contador de partículas.

\section{ATIVIDADES EXPERIMENTAIS}

\subsection{O mock-up e os sistemas de ventilação}

Os ensaios foram realizados em mock-up de 12 lugares, também instalado no Laboratório de Conforto Térmico e Qualidade do Ar do Departamento de Engenharia Mecânica da Escola Politécnica da USP, com manequins aquecidos simulando pessoas, conforme mostrado na Figura 7. Nas Figuras 8 e 9 são apresentadas representações esquemáticas dos sistemas de ventilação pelo teto (MV) e pelo piso (UFAD).
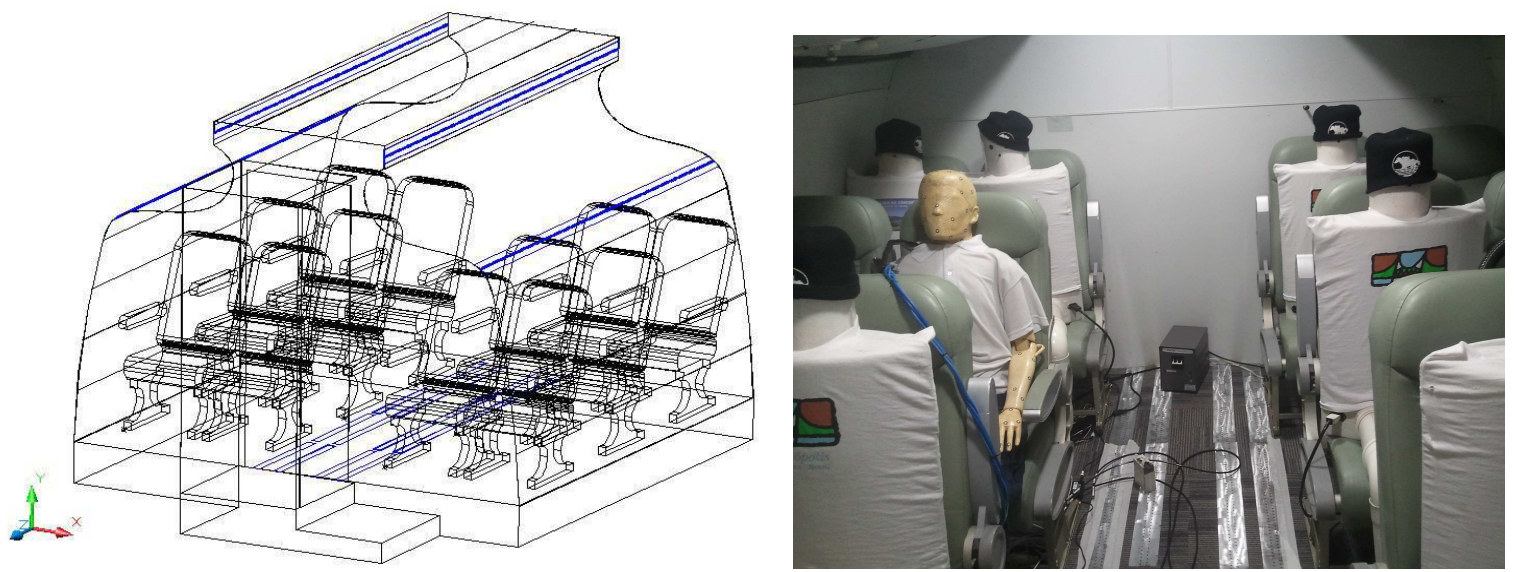

Figura 7. Vista interna do mock-up e dos manequins aquecidos.

\subsection{O gerador e o contador de partículas}

Com base nos requisitos estabelecidos por Conceição, 2012, foi escolhido o gerador de aerossol da TSI Modelo 3475 (Fig. 10), que produz partículas com diâmetro médio entre 0,1 a $8 \mu \mathrm{m}$, faixa esta que engloba a maior quantidade de partículas geradas em atividades expiratórias e pode produzir aerossol com concentração de $10^{11}$ partículas $/ \mathrm{m}^{3}$ [19].

O aerossol é gerado com DEHS (Di-etil-hexil-sebacato), que é um líquido com baixa taxa de evaporação e massa específica próxima à da água $\left(915 \mathrm{~kg} / \mathrm{m}^{3}\right)$ [20]. Acompanha o equipamento um monitor de partículas PAM 3475, instalado em série, que monitora o diâmetro médio das partículas geradas, bem como a sua concentração [19]. 


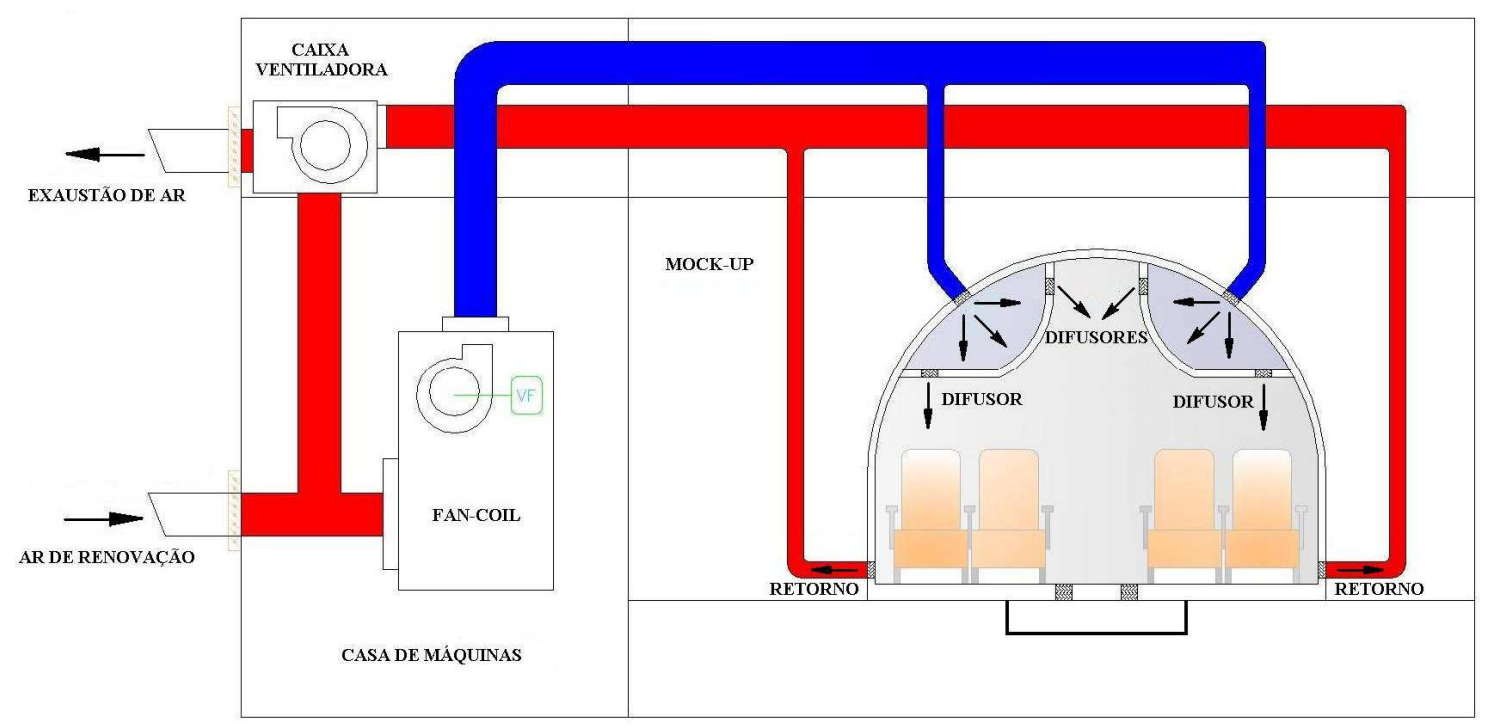

Figura 8. Sistema de ventilação pelo teto (MV).

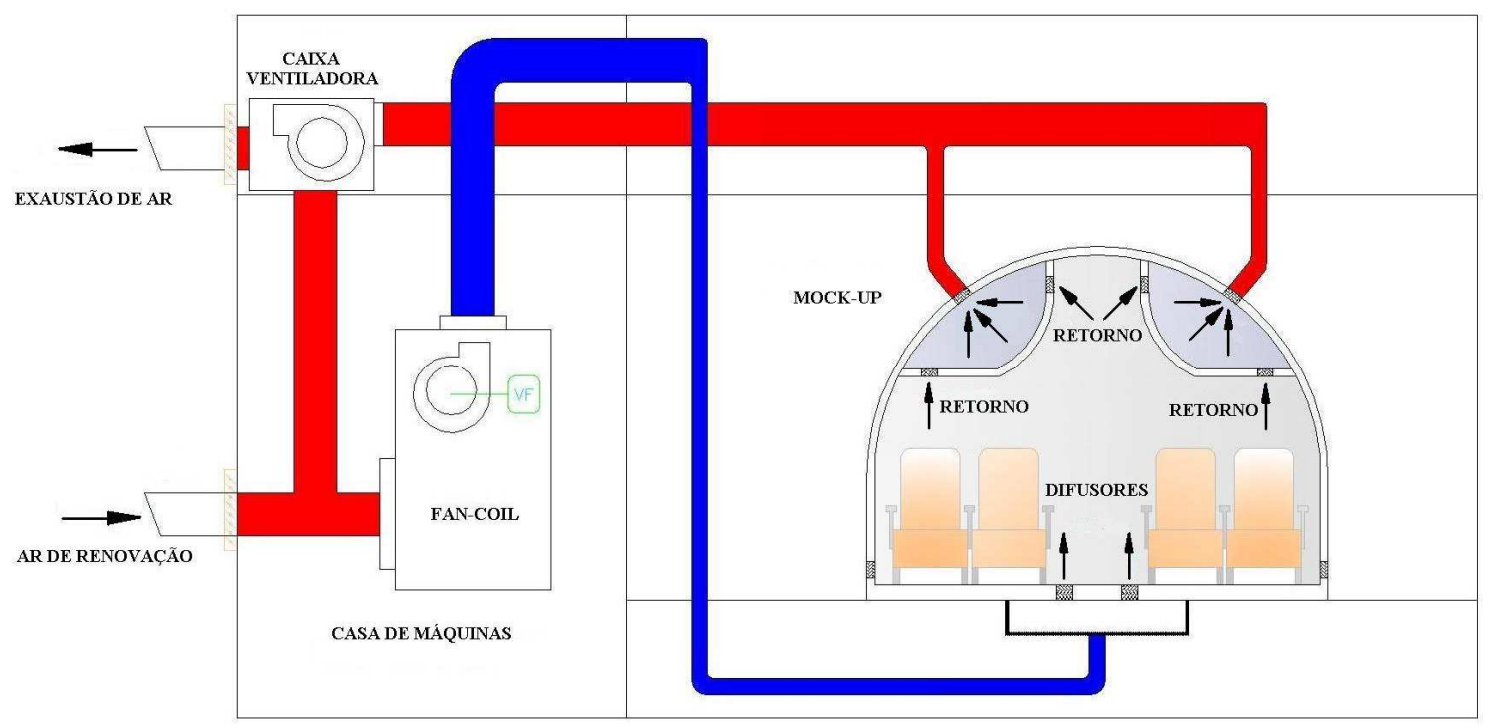

Figura 9. Sistema de ventilação pelo piso (UFAD).

O gerador opera com vazão de aerossol da ordem de $4 \mathrm{~L} / \mathrm{min}$, semelhante à vazão média de ar de inalação/exalação das pessoas [7]. Considerando que o diâmetro do tubo de saída de aerossol é da ordem de $10 \mathrm{~mm}$, a velocidade na saída esperada é de aproximadamente $1 \mathrm{~m} / \mathrm{s}$. É importante destacar que o gerador selecionado foi utilizado por outros pesquisadores em estudos correlatos, dentre eles Zhang et al., [5], Jones e Nicas, [21] e Conceição, [9].

A medição das partículas foi realizada com contador óptico da marca Met One (Fig.11), que possui 6 canais para a contagem de partículas na faixa de 1,0 a $10,0 \mu \mathrm{m}(1,0$ a 2,0 $\mu \mathrm{m}$, de 2,0 a $3,0 \mu \mathrm{m}$, de 3 a $5 \mu \mathrm{m}$, de 5 a $7 \mu \mathrm{m}$, de 7 a $10 \mu \mathrm{m}$, e maior do que $10 \mu \mathrm{m})$. Ou seja, o contador permite medir partículas na faixa gerada pelo gerador TSI modelo 3475 . 


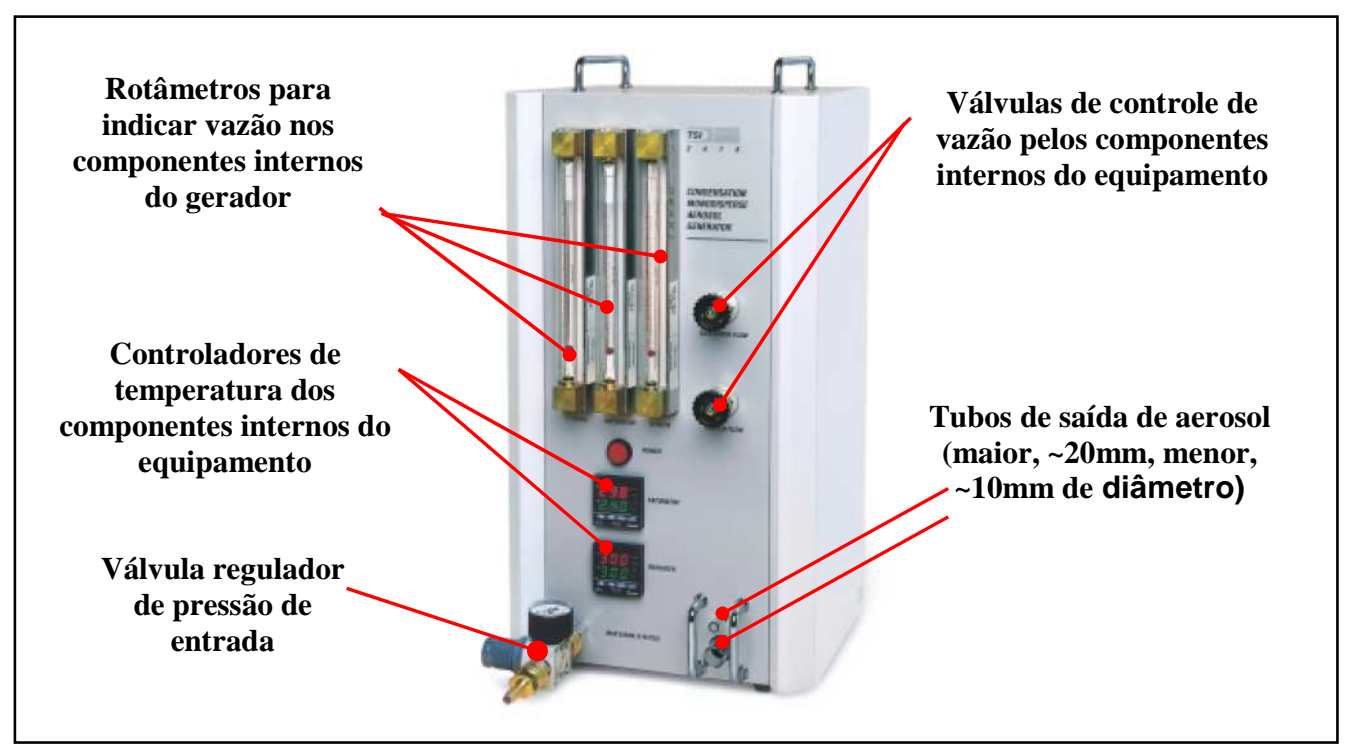

Figura 10. Gerador de aerossol TSI modelo 3475 [19] - imagem de catálogo.

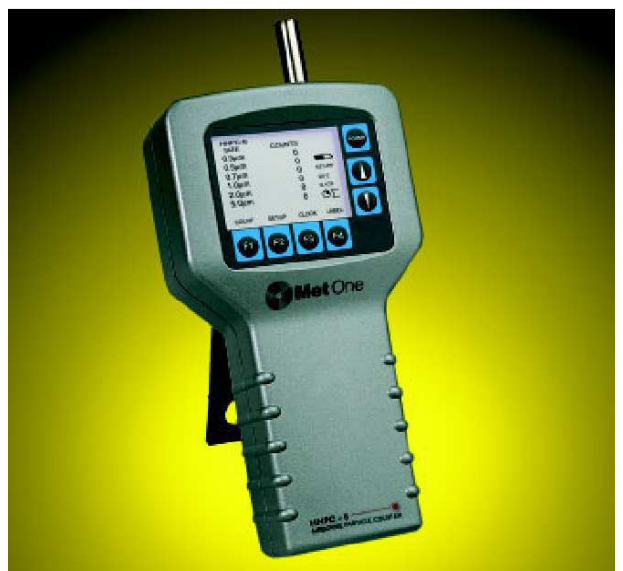

Figura 11. Contador de partículas da MetOne, modelo HHPC6 (Met One HHPC-6, 2010).

Este equipamento captura o ar do seu entorno por sucção promovida por uma bomba interna com vazão volumétrica de $0,1 \mathrm{cfm}(2,83 \mathrm{~L} / \mathrm{min}$.). A duração de cada amostragem pode ser definida entre $6 \mathrm{~s}$ e $10 \mathrm{~min}$, o que determina o volume total de ar amostrado em cada medição. Já o intervalo entre as amostragens pode ser definido entre $1 \mathrm{~s}$ e $1 \mathrm{~h}$. $\mathrm{O}$ tempo de duração mais adequado pode variar em função da aplicação específica e deve ser definido empiricamente.

O contador opera pelo principio de dispersão de um feixe luminoso (laser de diodo) ocasionada pela passagem das partículas aspiradas pela bomba. Um tubo coletor de partículas com diâmetro interno de $10 \mathrm{~mm}$ acompanha o contador de partículas (Fig, 11).

Segundo o manual deste equipamento, o erro de coincidência é da ordem $5 \%$ para concentrações de $2.10^{6} /$ pés $^{3}\left(7,0.10^{7} / \mathrm{m}^{3}\right)$ e a eficiência de contagem é de $50 \%$ para partículas de $0,3 \mu \mathrm{m}$ e de $100 \%$ para partículas maiores do que $0,45 \mu \mathrm{m}$. 


\subsection{Procedimento experimental}

Os ensaios foram realizados para temperatura de ar de insuflamento de $18^{\circ} \mathrm{C}$ com vazão de ar de $9,5 \mathrm{l} / \mathrm{s}$ por pessoa, que corresponde a $34 \mathrm{~m}^{3} / \mathrm{h}(20 \mathrm{cfm})$ por pessoa, conforme recomendado pela norma ASHRAE 161 [22], perfazendo vazão total de $408 \mathrm{~m}^{3} / \mathrm{h}$ (cabine com 12 pessoas). A vazão de ar foi determinada e monitorada a partir da medição da velocidade do ar nos dutos de retorno utilizando tubos de Pitot.

Seguindo procedimento adotado em cabine de aeronaves, no insuflamento pelo teto (MV) $60 \%$ da vazão de ar foi insuflada pelos difusores junto à fuselagem e $40 \%$ pelos difusores localizados na parte superior dos bagageiros. De modo similar, no insuflamento pelo piso (UFAD), $60 \%$ da vazão de exaustão deu-se pelas grelhas laterais de retorno junto à fuselagem e $40 \%$ pelas grelhas de retorno acima dos bagageiros (Figs. 8 e 9).

Para a verificação das condições do escoamento e de condições de ensaio em regime permanente, temperaturas e velocidades do ar no interior da cabine foram medidas com a utilização de termopares e anemômetros omnidirecionais, com incertezas de medição, respectivamente, de $\pm 0,5{ }^{\circ} \mathrm{C}$ e $\pm(0,02+0,02 \mathrm{~V}) \mathrm{m} / \mathrm{s}$, que atendem aos requisitos de precisão de equipamentos da norma ISO 7730 [23]. Os planos de medição das velocidades e temperaturas são apresentados na Figura 12.
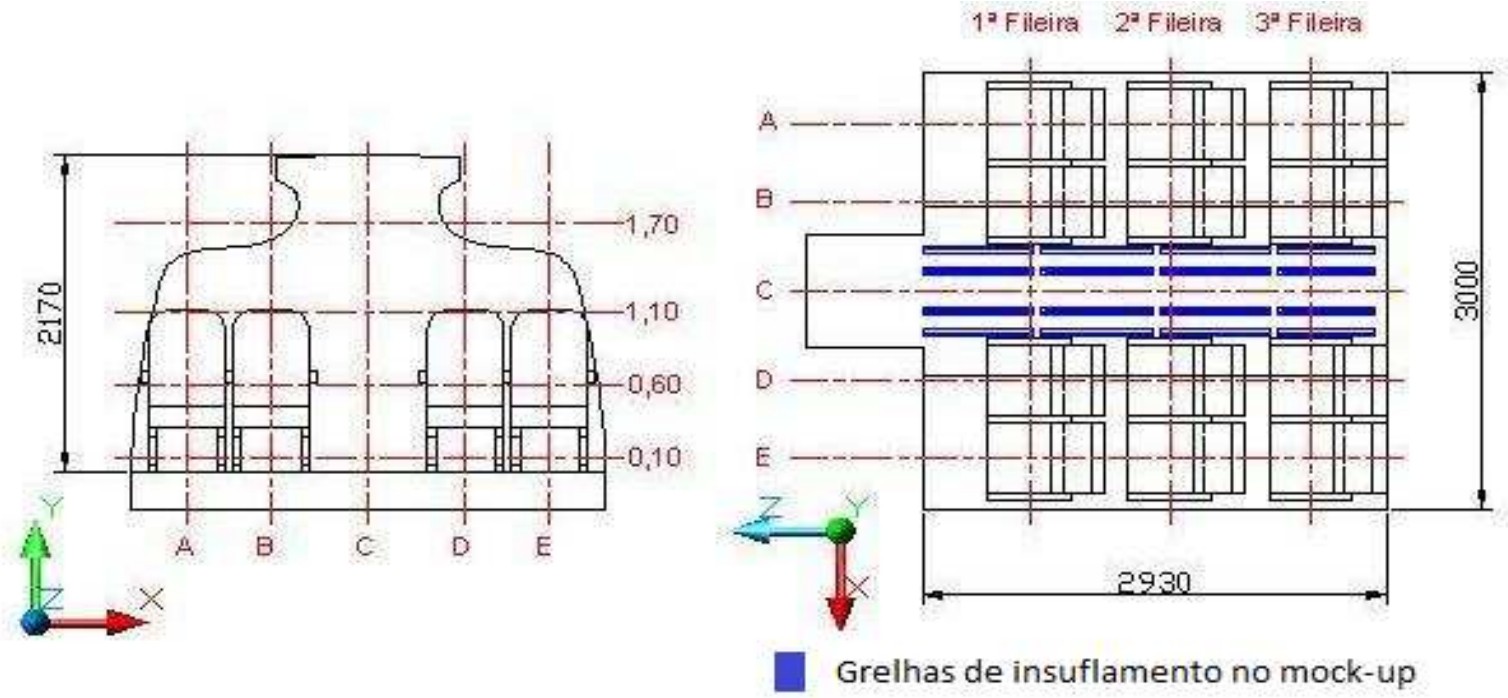

Figura 12. Planos de medição de temperaturas e de velocidades do ar na cabine.

Em todos os ensaios a temperatura das paredes foi mantida próxima à temperatura do ar da cabine. Este procedimento foi utilizado para não introduzir mais uma variável no estudo da contaminação aérea no presente trabalho.

A umidade relativa do ar foi mantida em 50\%. Em função de ter sido utilizado o DEHS na geração das partículas, que é um líquido com baixa taxa de evaporação, a umidade relativa do ar da cabine não deve influenciar significativamente na concentração de partículas medidas ao longo do experimento [5] e [9].

A exemplo do trabalho desenvolvido por Conceição, [9], o gerador de aerossol foi configurado para gerar partículas com tamanho médio de $4 \mu \mathrm{m} \pm 1 \mu \mathrm{m}$ e concentração em torno de $0,61.10 \% \mathrm{~cm}^{3}$. Esta concentração de partículas é da mesma ordem de grandeza da concentração de gotículas geradas em um espirro [24]. 
Tendo em vista os resultados obtidos por Wan et al., [4], que mostraram que um menor número de partículas foi disperso para outros assentos quando a injeção das partículas foi feita simulando passageiro junto à fuselagem em comparação com passageiro sentado em poltrona no centro do mock-up, no presente trabalho também foram realizados ensaios considerando estas duas possibilidades. Os pontos de injeção de aerossol, juntamente com os pontos de medição das partículas, são mostrados na Figura 13.

As medições de concentração de partículas foram realizadas na altura de $1,10 \mathrm{~m}$ a partir do piso (região de respiração), com medições realizadas após se atingir condições de regime permanente. Para evitar retorno das partículas pelo sistema de climatização, os ensaios foram realizados com $100 \%$ de ar de renovação.

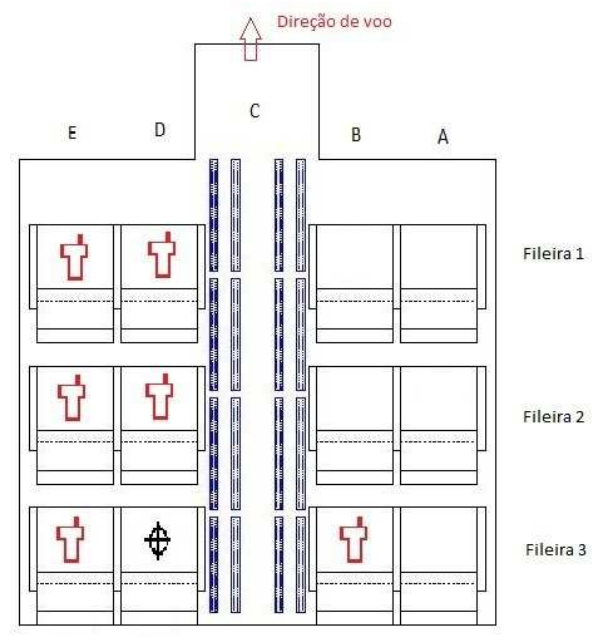

(a)

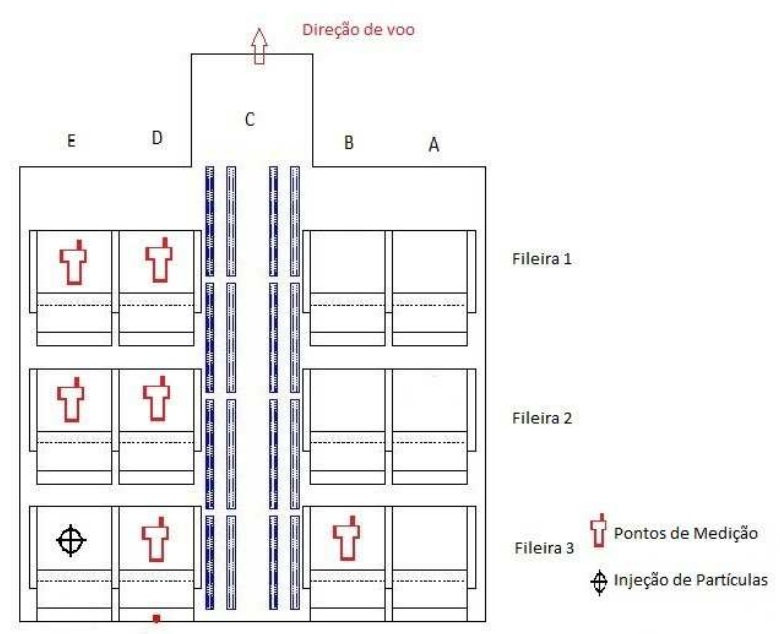

(b)

Figura 13. Pontos de injeção e medição de partículas no mock-up (a) injeção de partículas na poltrona 3D (b) injeção de partículas na poltrona 3E

\section{ANÁLISE DE RESULTADOS}

À título de ilustração, nas Figuras 14 e 15 são apresentados, respectivamente, os perfis de temperaturas e velocidades do ar obtidos ao longo da cabine (Fig. 12) para o sistema de ventilação pelo teto (MV) e pelo piso (UFAD). Verifica-se da análise dessas figuras que os perfis de temperatura e de velocidade do ar são completamente diferentes para cada sistema de ventilação, função do tipo de escoamento que resulta da forma como o ar é insuflado no ambiente.

Estes diferentes perfis de temperatura e velocidade do ar acarretam diferentes padrões de distribuição de partículas na cabine nos dois sistemas de ventilação, conforme pode ser verificado nas Figuras 16 e 17. 


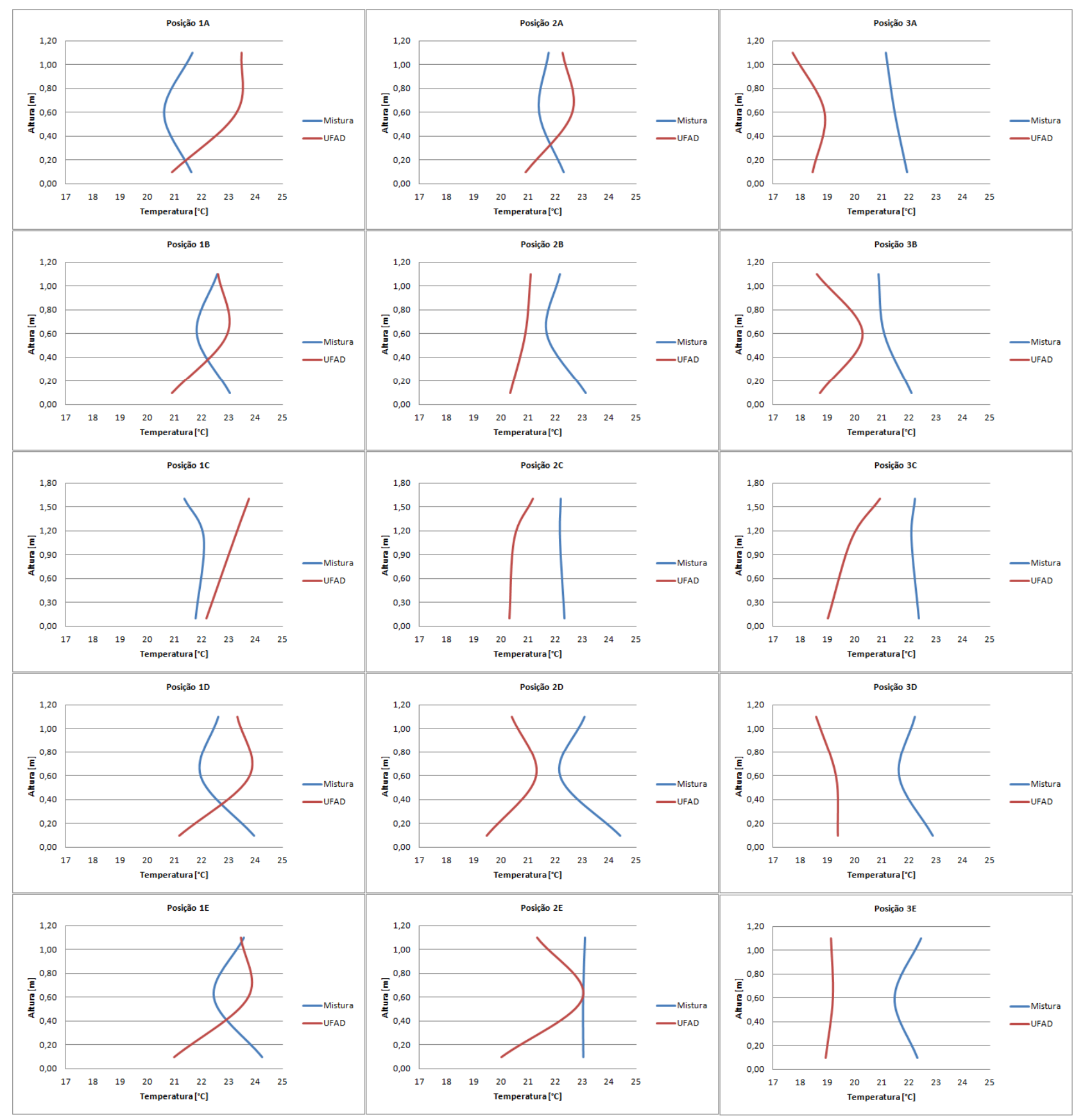

Figura 14. Perfis de temperatura do ar para sistema MV e sistema UFAD. 


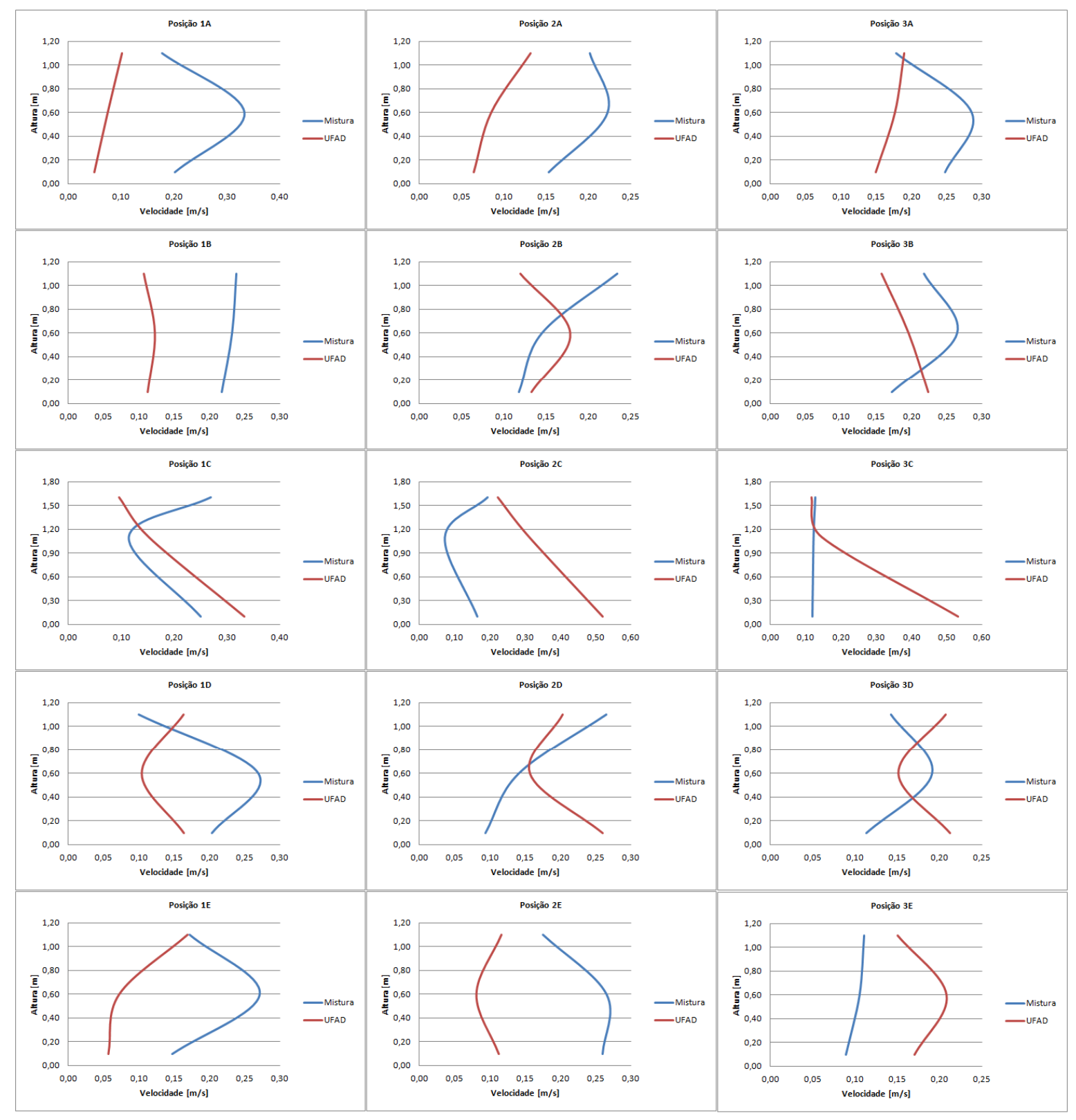

Figura 15. Perfis de velocidade do ar para sistema MV e sistema UFAD. 


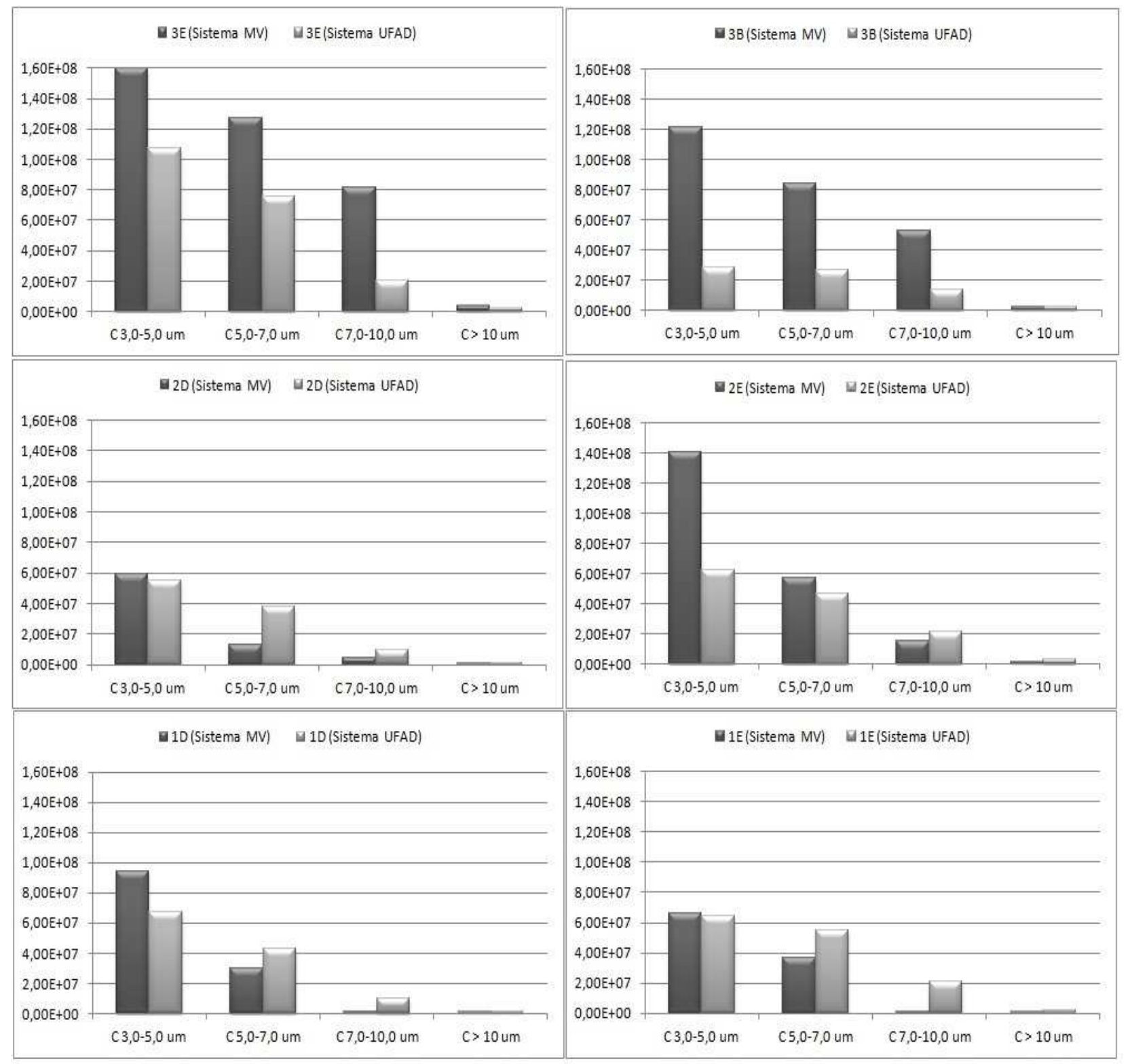

(*) No assento 3E, sistema MV, a concentração para tamanhos de partículas entre 3 e $5 \mu \mathrm{m}$ é de 3,50E+08 partículas $/ \mathrm{m}^{3}$, cujo valor extrapola a escala utilizada. Contudo, foi utilizada esta escala, pois uma escala maior não permitiria a visualização de quantidades menores de partículas de outros tamanhos e assentos.

Figura 16. Quadro comparativo de distribuição de partículas nos sistemas MV e UFAD com injeção de partículas na poltrona 3D 


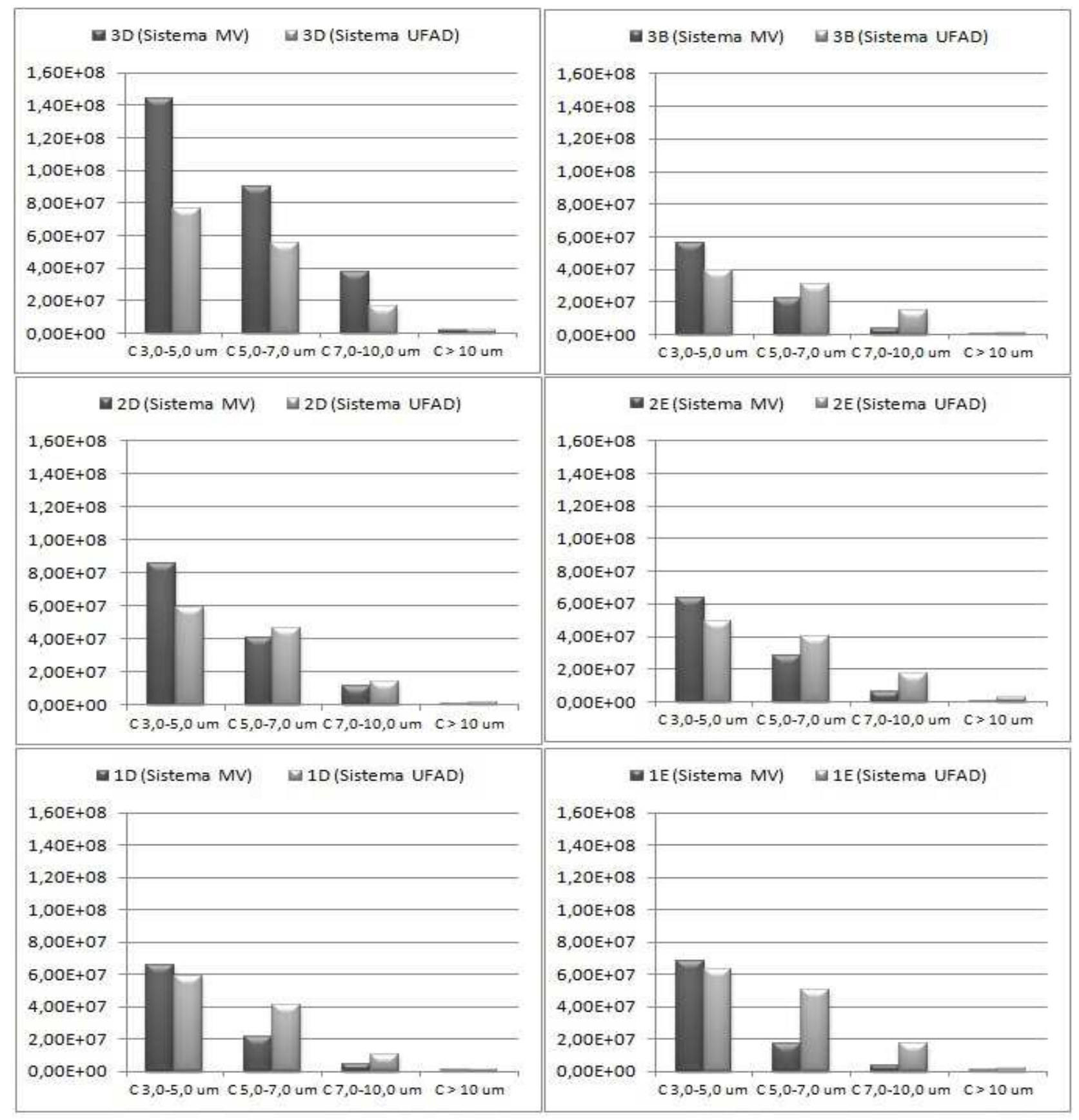

Figura 17. Quadro comparativo de distribuição de partículas nos sistemas MV e UFAD com injeção de partículas na poltrona $3 \mathrm{E}$

$\mathrm{Na}$ análise das Figuras 16 e 17 verifica-se que o sistema de ventilação pelo teto, MV, promove uma maior dispersão de partículas ao longo da cabine, função da característica de mistura desse tipo de sistema de ventilação. Além disso, verifica-se uma maior concentração de partículas na cabine no sistema de ventilação $\mathrm{MV}$, indicando uma menor eficiência na remoção das partículas da cabine nesse sistema de ventilação. Por outro lado, o sistema pelo piso, UFAD, apresentou uma maior eficiência na remoção de partículas, principalmente, na região próxima à injeção de partículas, com pequeno aumento na concentração de partículas maiores em regiões mais afastadas do ponto de injeção. Ou seja, o sistema UFAD permite um 
maior controle na remoção e dispersão de partículas e, com isto, um maior controle na dispersão de contaminantes no ar.

Além disto, a análise comparativa entre os resultados apresentados nas Figuras 16 e 17, ou seja, entre a injeção de partículas no assento 3D (junto ao corredor) e 3E (junto à fuselagem), respectivamente, mostra um aumento considerável (praticamente o dobro) na concentração de partículas no assento 3E, quando da injeção de partículas no assento 3D, em comparação à concentração de partículas no assento 3D, quando da injeção de partículas no assento 3E, quando da utilização de sistema MV. Ou seja, foram obtidos no presente trabalho resultados similares àqueles obtidos por Wan et al., [1], que mostram um menor número de partículas dispersas para outros assentos quando a injeção das partículas foi feita simulando passageiro junto à fuselagem em comparação com passageiro sentado em poltrona junto ao corredor do mock-up.

\section{CONCLUSÃO}

Com o desenvolvimento do presente trabalho verificou-se que a dispersão de partículas sofre grande influência do sistema de ventilação utilizado e do ponto de injeção de partículas. Tendo em vista as características dos sistemas de ventilação estudados, nota-se que o sistema UFAD apresenta grande potencial para a melhoria da qualidade do ar em cabines de aeronaves.

\section{REFERÊNCIAS}

[1] WAN, M. P., SZE TO, G.N., CHAO, C. Y. H., FANG, L. MELIKOV, A., Modeling the Fate of Expiratory Aerosols and the Associated Infection Risk in an Aircraft Cabin Environment', Aerosol Science and Technology, 43: 4, 322-343.(2009)

[2] OLSEN S.J., CHANG H.L., CHEUNG T.Y., Transmission of the severe acute respiratory syndrome on aircraft. New England Journal of Medicine, 349, pp. 24162422.(2003)

[3] MANGILI A., GENDREAU, M.A.,Transmission of infectious diseases during commercial air travel. Lancet; 365: 989-96 (2005)

[4] WAN, M.P., CHAO, C.Y.H., FANG, L., transmission characteristics of passengerexhaled droplets in a simulated air-cabin environment. Proceedings of Indoor Air (2005).

[5] ZHANG Z., CHEN X., MAZUNDAR S., ZHANG, T. CHEN, Q., Experimental and numerical investigation of airflow and contaminant transport in an airliner cabin mock-up. Building and Environment, doi:10.1016/j.buildenv.2008.01.012.(2008)

[6] SZE TO, G.N., WAN, M. P., Chao, C. Y. H., Fang, L. Melikov, A. 'Experimental Study of Dispersion and Deposition of Expiratory Aerosols in Aircraft Cabins and Impact on Infectious Disease Transmission', Aerosol Science and Technology, 43: 5, 466 - 485.(2009)

[7] YAN, W., ZHANG, Y., SUN, Y., LI, D., Experimental and CFD study of unsteady airborne pollutant transport within an aircraft cabin mock-up. Building and Environment 44 34-43.(2009)

[8] GUPTA, J.K., Lin, C.H., Chen, Q., 2011, Transport of expiratory droplets in an aircraft cabin, Indoor Air; 21: 3-11 (2011). 
[9] CONCEIÇÃO, S. T., Contaminação aérea em cabines climatizadas: Processo de avaliação e análise da influência de sistema de ventilação personalizado. Tese de Doutorado. Departamento de Engenharia Mecânica, Escola Politécnica da USP, São Paulo, 218p.(2012)

[10] HUNT, E.H., SPACE, D.R. "The Airplane Cabin Environment - Issues Pertaining to Flight Attendant Comfort", The Boeing Company, International In-flight Service Management Organization Conference, Montreal, Canada.(1994)

[11] GAO, N.P. NIU, J.L., PERINO, M. HEISELBERG,P., The airborne transmission of infection between flats in high-rise residential buildings: Tracer gas simulation, Building and Environment 43: 1805-1817.(2007).

[12] ZHANG T, CHEN X, MAZUNDAR S, ZHANG, T. CHEN, Q., Experimental and numerical investigation of airflow and contaminant transport in an airliner cabin mockup. Proceedings of the $10^{\text {th }}$ International Conference on Air Distribution in Rooms Roomvent 2007. Helsinki.,(2007).

[13] ZHANG T., CHEN Q., Novel air distribution systems for commercial aircraft cabins. Building and Environment (42):1675-1684., (2007).

[14] DLR,. DLR and Airbus flight-test new aircraft ventilation system. http://www.dlr.de/dlr/en/desktopdefault.aspx/tabid-10256/366 read-1664 . acessado em 05.04.2013.(2011).

[15] CHEN Q, GLICKSMAN L., System performance evaluation and design guidelines for displacement ventilation. Altanta, GA: ASHRAE.,(2003).

[16] BAUMAN F., DAILY A., Underfloor air distribution (UFAD) design guide. American Society of Heating, Refrigerating and Air-Conditioning Engineers, Inc., Atlanta., (2003)

[17] LEITE, B. C. C. , TRIBESS, A., Analysis of thermal comfort in an office environment with underfloor air supply in a tropical climate. HVAC\&R Research. 12(2): 215-229.,(2006)

[18] PEREIRA, M. L., GRAUDENZ, G., TRIBESS, A., MORAWSKA, L., Determination of particle concentration in the breathing zone for four different types of office ventilation systems. Building and Environment. 44: 904-911.,(2009).

[19] TSI, Particle Generator Model 3079. Manufacturer manual available in: www.atitest.com. Accessed on July, 2010.TSI, 2004, Model 3475 Condensation Monodisperse Aerosol Generator Instruction Manual, Revision E, TSI, outubro (2004).

[20] HINDS, W. C. Aerosol Technology. New York, John Wiley Sons, Inc.,(1999)

[21] JONES, R., NICAS, M., Experimental Determination of Supermicrometer Particle Fate Subsequent to a Point Release within a Room under Natural and Forced Mixing. Aerosol Science and Technology, 43: 9, 921-938.,(2009)

[22] ANSI/ASHRAE Standard 16, Air Quality Within Commercial Aircraft. American Society of Heating, Refrigerating and Air-Conditioning Engineers, Inc., Atlanta.,(2007).

[23] ISO 7726. Thermal environments - Instruments and methods for measuring physical quantities, International Organization for Standardization, Geneva, (1998).

[24] DUGUID, J. P., The Size and the Duration of Air-Carriage of Respiratory Droplets and Droplet-Nuclei, J. Hyg. 44:471-479.,(1946). 